\title{
Recent progress on monolithic fiber amplifiers for next generation of gravitational wave detectors
}

Wellmann, Felix, Booker, Phillip, Hochheim, Sven, Theeg, Thomas, de Varona, Omar, et al.

Felix Wellmann, Phillip Booker, Sven Hochheim, Thomas Theeg, Omar de Varona, Willy Fittkau, Ludger Overmeyer, Michael Steinke, Peter Weßels, Jörg Neumann, Dietmar Kracht, "Recent progress on monolithic fiber amplifiers for next generation of gravitational wave detectors," Proc. SPIE 10512, Fiber Lasers XV: Technology and Systems, 105120I (26 February 2018); doi: 10.1117/12.2289919

SPIE. Event: SPIE LASE, 2018, San Francisco, California, United States 


\title{
Recent Progress on Monolithic Fiber Amplifiers for Next Generation of Gravitational Wave Detectors
}

\author{
Felix Wellmann ${ }^{1,{ }^{*}}$, Phillip Booker ${ }^{1}$, Sven Hochheim ${ }^{1}$, Thomas Theeg ${ }^{1}$, Omar de Varona ${ }^{1}$, \\ Willy Fittkau $^{1}$, Ludger Overmeyer ${ }^{1,3}$, Michael Steink $e^{1,2}$, Peter Weßels ${ }^{1,2}$, Jörg Neumann ${ }^{1,2}$, \\ Dietmar Kracht ${ }^{1,2}$ \\ 1. Laser Zentrum Hannover e.V., Hollerithallee 8, D-30419 Hannover, Germany \\ 2. Centre for Quantum-Engineering and Space-Time Research - QUEST, Welfengarten 1, D-30167 Hannover, Germany \\ 3. Institut für Transport- und Automatisierungstechnik, Leibniz Universität Hannover, An der Universität 2, 30823 Garbsen, Germany \\ *f.wellmann@lzh.de
}

Single-frequency fiber amplifiers in MOPA configuration operating at $1064 \mathrm{~nm}\left(\mathrm{Yb}^{3+}\right)$ and around $1550 \mathrm{~nm}$ $\left(\mathrm{Er}^{3+}\right.$ or $\left.\mathrm{Er}^{3+}: \mathrm{Yb}^{3+}\right)$ are promising candidates to fulfill the challenging requirements of laser sources of the next generation of interferometric gravitational wave detectors (GWDs). Most probably, the next generation of GWDs is going to operate not only at $1064 \mathrm{~nm}$ but also at $1550 \mathrm{~nm}$ to cover a broader range of frequencies in which gravitational waves are detectable. We developed an engineering fiber amplifier prototype at $1064 \mathrm{~nm}$ emitting $215 \mathrm{~W}$ of linearly-polarized light in the $\mathrm{TEM}_{00}$ mode. The system consists of three modules: the seed source, the pre-amplifier, and the main amplifier. The modular design ensures reliable long-term operation, decreases system complexity and simplifies repairing and maintenance procedures. It also allows for the future integration of upgraded fiber amplifier systems without excessive downtimes. We also developed and characterized a fiber amplifier prototype at around $1550 \mathrm{~nm}$ that emits $100 \mathrm{~W}$ of linearly-polarized light in the $\mathrm{TEM}_{00}$ mode. This prototype uses an $\mathrm{E}^{3+}: \mathrm{Yb}^{3+}$ codoped fiber that is pumped off-resonant at $940 \mathrm{~nm}$. The off-resonant pumping scheme improves the $\mathrm{Yb}^{3+}$-to-Er ${ }^{3+}$ energy transfer and prevents excessive generation of $\mathrm{Yb}^{3+}$-ASE.

Keywords: gravitational waves, monolithic fiber amplifier, single-frequency

\section{INTRODUCTION}

At the beginning of 2016, the first direct observation of gravitational waves by an interferometric gravitational wave detector (GWD) has been reported [1]. Several further detections, partly confirmed by independent observations in the electromagnetic spectrum [2], led to an increased interest in a new generation of GWDs with increased sensitivity. Several design studies have been compiled [3,4] and propose GWDs that are particularly more sensitive at lower frequencies, which would open a new range of detectable astrophysical events. Two different design approaches are discussed: Operation at room temperature with laser systems at $1064 \mathrm{~nm}$ or operation at cryogenic temperatures with laser systems at around $1550 \mathrm{~nm}$. Cryogenic cooling of the interferometer optics is considered as a promising strategy to decrease thermal noise and increase the instrument's sensitivity, in particular at low frequencies. However, if fused silica is used as substrate material the mechanical loss increases with decreasing temperatures up to a point where the overall instrument sensitivity is affected [5]. Instead of using fused silica as substrate material, crystalline silicon is discussed because of its better mechanical properties at cryogenic temperatures. However, silicon is only transparent for wavelengths above $1.4 \mu \mathrm{m}$. Therefore, laser and amplifier operating around $1550 \mathrm{~nm}$ are very promising for nextgeneration cryogenic GWDs.

Current state-of-the-art GWDs, i.e., the US-based LIGO detectors, use solid-state injection-locked Nd:YAG ring oscillators as high-power laser sources. These lasers fulfill the power output and noise requirements but have certain disadvantages regarding usability, maintenance procedures and further power scaling. At increased output power levels, these lasers require complex cooling strategies and tend to increased thermal lensing, which leads to instabilities and reduction of beam quality. System inherently, the output power cannot be freely chosen because the thermal lensing is part of the resonator design and is highly optimized for a pre-defined working point. The complexity of the solid-state

Fiber Lasers XV: Technology and Systems, edited by Ingmar Hartl, Adrian L. Carter, Proc. of SPIE

Vol. 10512, 105120I · C 2018 SPIE · CCC code: 0277-786X/18/\$18 · doi: 10.1117/12.2289919 
laser system and its cooling system makes maintenance and repair more difficult. The fiber technology has been identified as a promising alternative technology to overcome limitations of the current laser systems. Fiber amplifiers enable very high output power levels and fulfill GWD requirements regarding low noise levels and exceptional beam quality (above $90 \% \mathrm{TEM}_{00}$ content). In addition to its optical properties, fiber amplifiers are less affected by environmental condition changes and produce lower and better distributed heat load, which make the system less complex. The reduced system complexity simplifies maintenance procedures, components upgrades, and repair. Therefore, high power monolithic fiber amplifier for $1064 \mathrm{~nm}$ and around $1550 \mathrm{~nm}$ that emit the linearly-polarized fundamental $\mathrm{TEM}_{00}$ mode are promising candidates and are thus further investigated.

In recent years, several monolithic single-frequency fiber amplifiers have been developed that demonstrated the power scalability of such devices. In 2008 Mermelstein et al. showed $194 \mathrm{~W}$ output power [6], in $2013 \mathrm{Ma}$ et al. up to $332 \mathrm{~W}$ output power [7] and recently Huang et al. demonstrated an output power of $414 \mathrm{~W}$ [8]. These amplifiers used $\mathrm{Yb}^{3+}$-doped large-mode-area (LMA) fibers to increase the threshold of stimulated Brillouin scattering (SBS), which is one of the power scaling limitations of single-frequency fiber amplifiers. We developed a fiber amplifier prototype particularly for the use in GWDs and characterized it according to their requirements. We demonstrated, besides the power scaling capabilities, the preservation of low noise properties and excellent beam quality. Our quasi-monolithic fiber amplifier system operating at $1064 \mathrm{~nm}$ delivers an output power of up to $215 \mathrm{~W}$ [9,11], without the onset of SBS. The setup consists of a narrow linewidth seed source with $2 \mathrm{~W}$ output power that is afterwards amplified in two fiber amplifier stages to $25 \mathrm{~W}$ and then to $215 \mathrm{~W}$. Both amplification stages use double-clad (DC) $\mathrm{Yb}^{3+}$-doped LMA fibers. Currently, we focused our work on an increased reliable and usable engineering fiber amplifier prototype.

We also developed a fiber amplifier prototype at $1550 \mathrm{~nm} \mathrm{[10]} \mathrm{that} \mathrm{shows} \mathrm{promising} \mathrm{properties} \mathrm{for} \mathrm{applications} \mathrm{in}$ next-generation cryogenic GWDs. As an active medium, an $\mathrm{Yb}^{3+}: \mathrm{Er}^{3+}$ codoped LMA fiber has been used. The amplifier was pumped off-resonantly at $940 \mathrm{~nm}$ to reduce the $\mathrm{Yb}^{3+}$-band ASE and to enable higher output power levels. The prototype obtains a maximum output power level of $100 \mathrm{~W}$ in the linearly polarized fundamental $\mathrm{TEM}_{00}$ mode. Currently, the system is only limited by the available pump power.

\section{SINGLE-FREQUENCY HIGH POWER FIBER AMPLIFIER AT 1064 NM}

Our fiber amplifier engineering prototype (c.f. Figure 1) consists of three individual modules, the seed and fiber incoupling stage, the first (pre-amplifier) amplifier stage and the second (high-power) amplifier stage. The modular design improves the usability, maintenance, and repair. Each module is separated from the other to give the system a more explicit arrangement, which improves error diagnostics procedures. Besides, in case of a module failure, a redundant module can replace the defective stage. This way only one or two splices between polarization maintaining (PM) fibers and two or four splices between multimode pump fibers need to be re-done. The design also enables easy replacement of a module by an upgraded version. Therefore, expensive downtimes because of laser failure or maintenance can be kept at a minimum.

The first stage comprises a non-planar ring oscillator (NPRO) as seed laser, an optical isolator to protect the seed source from back-reflections, and optics to mode-match the beam into a PM 10/125 fiber. The first stage has the necessary equipment to monitor power fluctuations and variations of the polarization. In case of a seed source failure, the NPRO can easily be replaced, and its alignment to the other optical components is mechanically kept equally to improve realignment procedures and keep laser downtime at a minimum. The module provides $1.6 \mathrm{~W}$ out of the fiber core, which is sufficient to fully saturate the first amplifier stage. The particular NPRO seed source technology has been proven over the last decade to show exceptional (power and frequency) noise properties and reliability. However, its output power is limited to a maximum of $2 \mathrm{~W}$, which is not sufficient for the use in GWDs; therefore, the power is amplified in two subsequent amplification modules. The first amplifier stage uses a $\mathrm{Yb}^{3+}$-doped 10/125 PM fiber that is pumped in a counter-propagating configuration. This configuration increases the SBS threshold by a more advantageous temperature distribution along the fiber [13]. Any residual pump light is removed by an in-house fabricated cladding light stripper (CLS) that relies on a microstructured cladding [18]. The seed signal is amplified by the first amplifier stage to a maximum power of $25 \mathrm{~W}$. The amplifier is protected from backward propagating light coming from the second amplifier stage by an optical isolator. The second amplifier stage is seeded by the pre-amplifier and is also counter-propagating pumped. The amplifier is capable of delivering $215 \mathrm{~W}$ of output power out of a PLMA Yb ${ }^{3+}$-doped $25 / 250$ fiber. A polarization extinction ratio (PER) of $18 \mathrm{~dB}$ has been obtained by the integration of the pump combiner, the CLS and the 
mode field adapter (MFA) directly in the active fiber. Therefore, additional splices have been avoided, which are usually the reason for a reduced PER. The four pump diodes, each emit a maximum power of $100 \mathrm{~W}$ at $976 \mathrm{~nm}$, are operated below their maximum output power to compensate for diode output power degradation or even complete failure of one diode. Additionally, the amplifier stage is equipped with diagnostics to analyze power fluctuations and to analyze the beam profile with an IR camera.

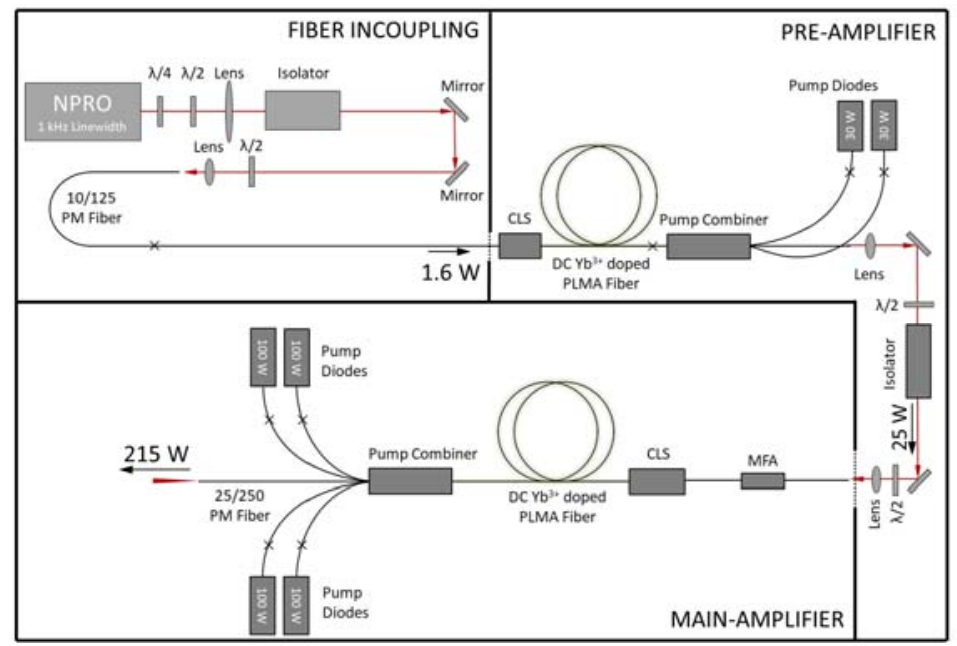

Figure 1: Schematic of the engineering prototype of a fiber amplifier at $1064 \mathrm{~nm}$ for the next generation of GWDs. The system consists of three stages: First stage: NPRO as a seed source, free space optics for mode-matching into the PM-10/125 fiber and an isolator for seed protection. This stage delivers $1.6 \mathrm{~W}$ at $1064 \mathrm{~nm}$. Second stage: The pre-amplifier stage consists of a monolithic fiber amplifier that uses an $\mathrm{Yb}^{3+}$-doped PM 10/125 fiber, which is pumped at $976 \mathrm{~nm}$. This stage delivers $25 \mathrm{~W}$ of output power and is protected by a high power isolator. Third stage: The final stage uses a $\mathrm{Yb}^{3+}$-doped $\mathrm{PM}^{\mathrm{N}}$ $25 / 250$ that is also pumped at $976 \mathrm{~nm}$ and is capable of delivering $215 \mathrm{~W}$ output power. CLS: Cladding light stripper; MFA: Mode field adapter.

The amplifier prototype system has been characterized regarding the requirements of GWDs. The beam quality, i.e., the fundamental $\mathrm{TEM}_{00}$ mode content was analyzed with a non-confocal scanning ring cavity as described in [14]. The eigenmodes of such a ring cavity correspond to TEM modes of the same order. Thus, by scanning such a cavity over a free spectral range allows the calculation of the relative content in the $\mathrm{TEM}_{00}$ mode and the higher order mode content. Figure 2 (a) shows the normalized mode scan at an output power of $120 \mathrm{~W}$ which corresponds to a fundamental mode content of 97-98\%. Figure 2 (b) shows the fundamental mode content and the measured PER for different output power levels.

(a)

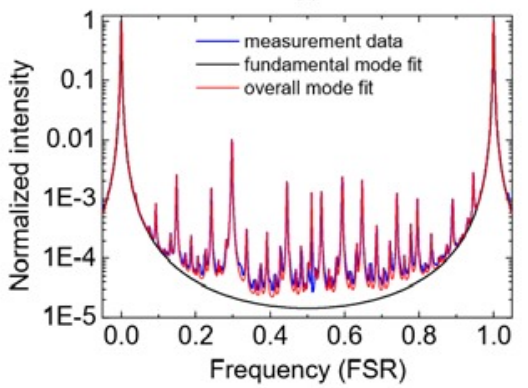

(b)

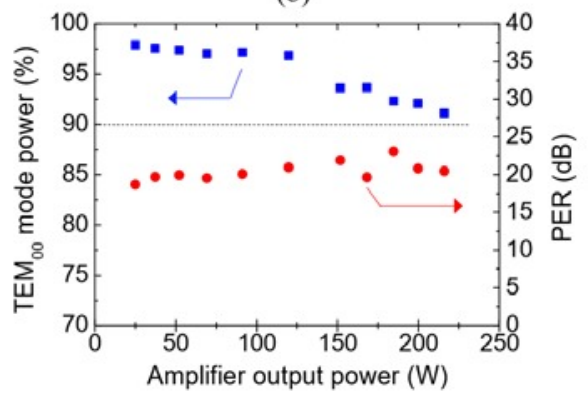

Figure 2: (a) Fundamental mode content measurement of the second amplifier stage at $120 \mathrm{~W}$ output power using a scanning ring cavity. (b) The fundamental mode content was calculated to be in the range of 97-98\%. At higher output power levels the $\mathrm{TEM}_{00}$ mode content decreases to $91 \%$. The measured PER remains above $18 \mathrm{~dB}$ over the entire output power range. [9] 


\section{SINGLE-FREQUENCY HIGH POWER FIBER AMPLIFIER AROUND 1550 NM}

Light amplification at $1550 \mathrm{~nm}$ in fiber amplifiers is more challenging compared to the $1064 \mathrm{~nm}$ fiber amplifier technology because of the properties of the used dopant $\mathrm{Er}^{3+}$. The absorption cross-section of $\mathrm{Er}^{3+}$ at the commonly used pump wavelength of $976 \mathrm{~nm}$, where pump diodes with very high output power are commercially available, is ten times lower than of $\mathrm{Yb}^{3+}$. Thus, amplifiers with sufficient pump light absorption require longer active fibers, which limit the output power by SBS or increased doping concentration, which is limited by the tendency of $\mathrm{Er}^{3+}$ to cluster during the fabrication process. The use of $\mathrm{Yb}^{3+}$ as a co-dopant enhances the pump light absorption at $976 \mathrm{~nm}$. In $\mathrm{Er}^{3+}: \mathrm{Yb}^{3+} \mathrm{codoped}$ fiber amplifiers the pump energy is mainly absorbed by the $\mathrm{Yb}^{3+}$-ions and transferred to $\mathrm{Er}^{3+}$ via a resonant energy transfer, i.e. a dipole-dipole interaction. However, such codoped amplifiers pumped at $976 \mathrm{~nm}$ generate excessive amounts of $\mathrm{Yb}^{3+}$-ASE, which can lead to self-lasing at high pump power levels. The reason for excessive $\mathrm{Yb}^{3+}-\mathrm{ASE}^{3}$ and self-lasing is the remaining energy that is not transferred to the $\mathrm{Er}^{3+}$ because of a limited and insufficient $\mathrm{Yb}^{3+}-\mathrm{to}^{-\mathrm{Er}^{3+}}$ energy transfer rate.

Recently, it has been demonstrated [15] that it is very promising to pump $\mathrm{Er}^{3+}: \mathrm{Yb}^{3+}$ codoped fibers off-resonantly at around $940 \mathrm{~nm}$. Pumping at this wavelength strongly reduces the $\mathrm{Yb}^{3+}$-ASE generation. The off-resonant pumping reduces the $\mathrm{Yb}^{3+}$ inversion because of the lower absorption cross section of $\mathrm{Yb}^{3+}$ at $940 \mathrm{~nm}$ and results in less $\mathrm{Yb}^{3+}$-ASE but at the expense of a slightly longer active fiber. The scheme also has the advantage of having the pump absorption and therefore the gain more homogeneously distributed along the fiber. The better gain distribution simplifies thermal management and thus reduces the complexity of the overall design. Because of the described advantages of this pumping scheme we developed a monolithic fiber amplifier at $1556 \mathrm{~nm}$ [10]. The prototype amplifier demonstrated over $100 \mathrm{~W}$ of output power at $1556 \mathrm{~nm}$ and was characterized aiming to its potential use in the third generation of GWDs.

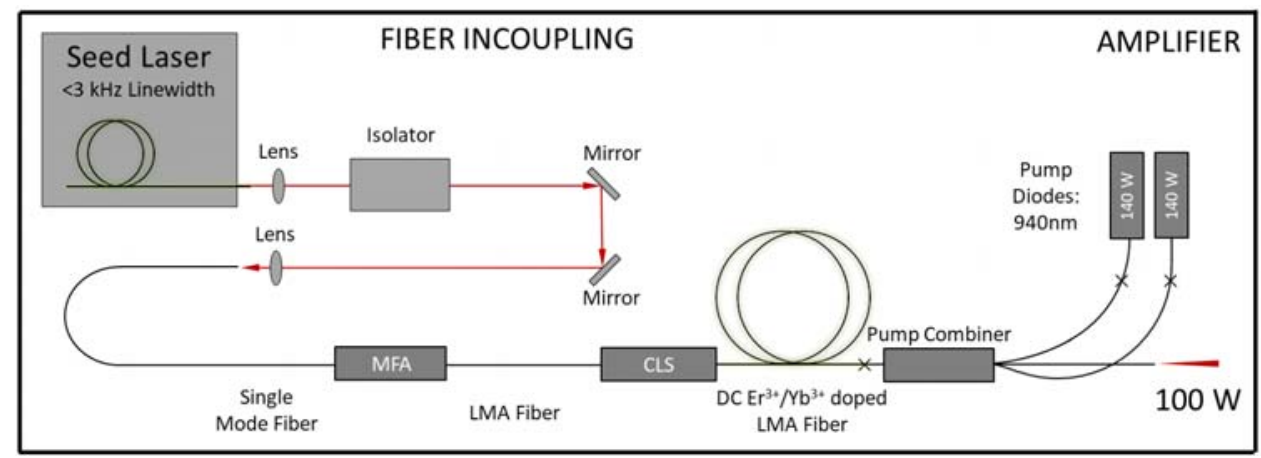

Figure 3: Fiber amplifier schematic for the operational wavelength of $1550 \mathrm{~nm}$. The setup consists of a $2 \mathrm{~W}$ seed source with an integrated internal pre-amplifier that represents the first amplification stage. This stage is protected by an isolator from backward propagating light. The seed light was mode-matched into the input fiber of the second amplifier stage. Because of flexibility and reusability reasons the preamplifier was not spliced directly to the input fiber of the second amplifier stage. Second stage: For beam quality reasons, the seed beam is coupled into a single mode fiber that is connected via a mode field adapter (MFA) to the LMA fiber of the amplifier. The double-clad $\mathrm{Er}^{3+}: \mathrm{Yb}^{3+}$ fiber is counter-propagation pumped to suppress SBS. Residual pump light is removed by a cladding light stripper (CLS).

Figure 3 shows the setup of the $1550 \mathrm{~nm}$ fiber amplifier prototype. A commercial single-frequency (kHz linewidth) fiber laser at $1556 \mathrm{~nm}$ with an internal pre-amplifier that provides a maximum output power of $2 \mathrm{~W}$ is used as a seed source. Although we could have spliced the seed module directly to the input fiber of the main amplifier, we free-space modematched the seed light into the input fiber for reasons of simple power monitoring, flexibility, and reusability. The input fiber is single-mode and is attached to an LMA 25/300 fiber via an in-house made mode field adapter (MFA). At a maximum pump power of $250 \mathrm{~W}$ a maximum output power of approximately $111 \mathrm{~W}$ at $1556 \mathrm{~nm}$ has been obtained with an efficiency of around $46 \%$ (c.f. Figure 4 a)). A fundamental mode content of $95 \%$ and a PER of $13 \mathrm{~dB}$ was obtained. That results in approximately $100 \mathrm{~W}$ of linear polarized $\mathrm{TEM}_{00}$ power. 

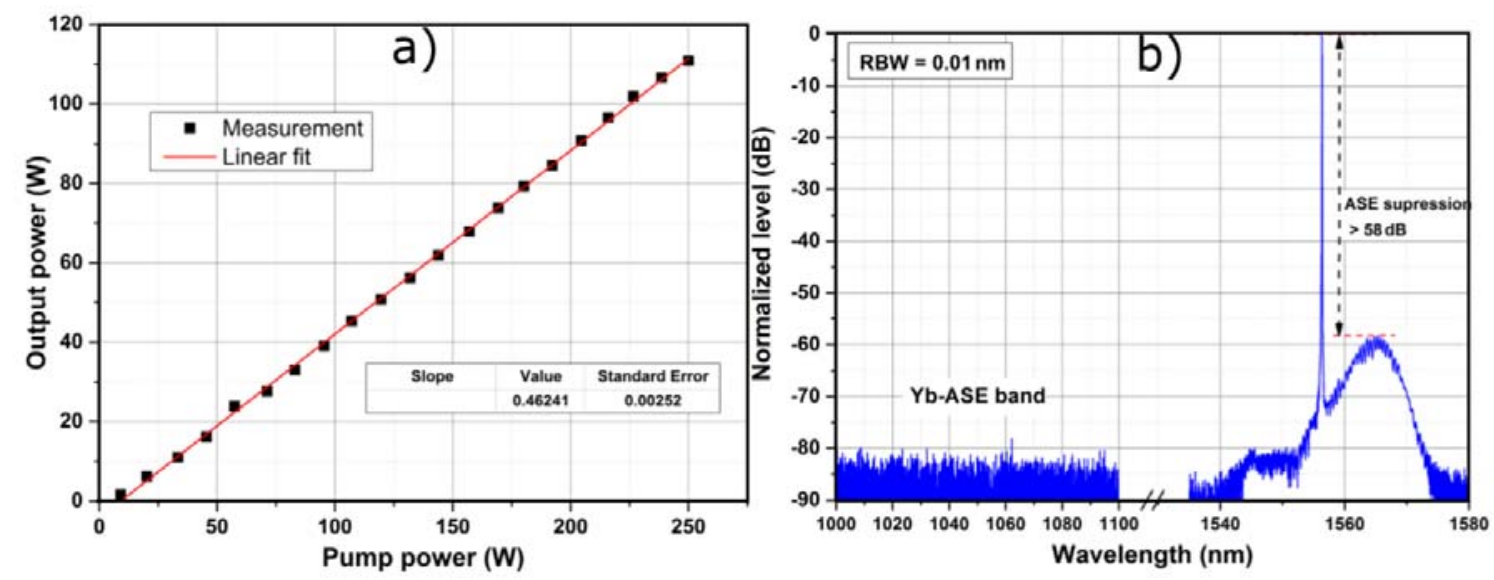

Figure 4: a) The slope shows an optical to optical efficiency of the fiber amplifier of approximately $46 \%$. The output power was limited by the available pump power and neither by thermal effects nor SBS. b) The optical spectrum shows an $\mathrm{Er}^{3+}-\mathrm{ASE}$ suppression of $58 \mathrm{~dB}$ and no signs of $\mathrm{Yb}^{3+}$-ASE. [10]

Figure $4 \mathrm{~b}$ ) shows the optical spectrum at an output power level of $111 \mathrm{~W}$ around the $\mathrm{Yb}^{3+}$-and $\mathrm{Er}^{3+}$-ASE band. No signs of $\mathrm{Yb}^{3+}$-ASE can be found within the spectrum. The $\mathrm{Er}^{3+}$-ASE was $58 \mathrm{~dB}$ below the signal level (measured with a resolution bandwidth of $10 \mathrm{pm}$ ). Besides the single frequency operation, a sufficient laser noise characteristic such as low relative power noise (RPN) are required for the operation in a GWD. Figure 5 shows the relative power noise spectral density at power levels of $2 \mathrm{~W}$ (seed only), $55 \mathrm{~W}$ and $110 \mathrm{~W}$, respectively. It is shown that the RPN is lowered at frequencies below $1 \mathrm{kHz}$ which is probably because of the damped high pass caused by the amplification process $[10,17]$. At higher frequencies of up to $100 \mathrm{kHz}$, the RPN of the amplifier is increased in comparison with the RPN of the seed source. The spectrum also shows some features that only occurred while pumping the amplifier with two laser diodes. Figure 5, black curve $(55 \mathrm{~W})$, shows an RPN measurement when pumped with one laser diode only. The operation of power levels above $55 \mathrm{~W}$ required the second pump diode and presumably is the reason for additional features within the noise spectrum which are caused by cross-coupling light between the pump ports of the combiner $[10]$.

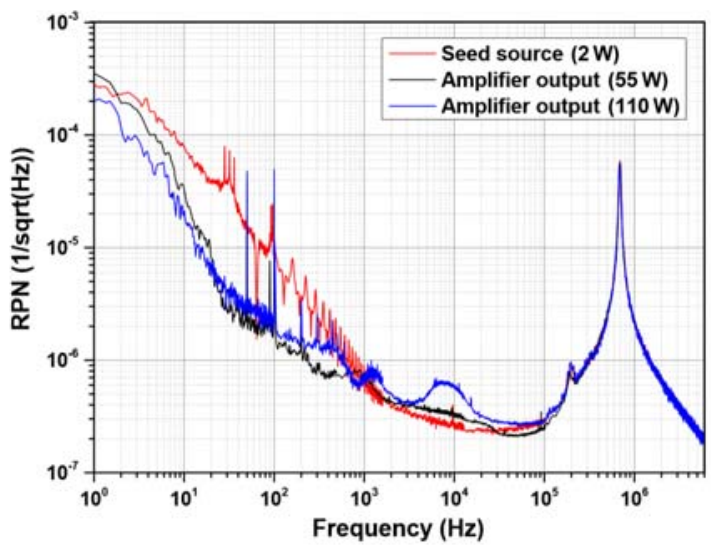

Figure 5: Relative power noise (RPN) measurements measured at $2 \mathrm{~W}$ (seed only), $55 \mathrm{~W}$ and $110 \mathrm{~W}$ output power. [10] 


\section{CONCLUSIONS}

Single-frequency fiber amplifiers in MOPA configuration are very promising laser sources to address the challenging requirements of next-generation GWDs. Monolithic systems can be compact, reliable, easy to operate, and simple to maintain. We developed a monolithic $\mathrm{Yb}^{3+}$-doped fiber amplifier using a step-index fiber with a $25 \mu \mathrm{m}$ core. The fiber amplifier system is capable of delivering $215 \mathrm{~W}$ of output power with a $\mathrm{TEM}_{00}$ mode content of more than $90 \%$ and a PER above $18 \mathrm{~dB}$. This system demonstrated that fiber amplifiers are capable of competing and outperform state-of-the-art solid-state laser system currently used in GWDs.

We also developed a monolithic $\mathrm{Er}^{3+}: \mathrm{Yb}^{3+}$ codoped fiber amplifier that is off-resonantly pumped at $940 \mathrm{~nm}$. The offresonant pumping enabled strong $\mathrm{Yb}^{3+}$-ASE suppression and the maximum output power was only limited by available pump power. A total output power of $100 \mathrm{~W}$ in a polarized $\mathrm{TEM}_{00}$ mode was achieved. The measured intensity and frequency noise of the amplifier output comply with the requirements of GWDs regarding the pre-stabilized laser system.

\section{ACKNOWLEDGMENTS}

The authors would like to thank Prof. Willke of the Albert-Einstein-Institut Hannover for the successful and on-going cooperation in the field of single-frequency laser systems for GWDs.

Funding was provided by the European Commission Seventh Framework Program (FP-PEOPLE-2013-ITN) (606176) and the Max Plank Institute for Gravitational Physics.

\section{REFERENCES}

[1] B.P. Abbott et al., LIGO Scientific Collaboration and Virgo Collaboration, "Observation of Gravitational Waves from a Binary Hole Merger,” Phys. Rev. Lett. 116 (6), 061102 (2016).

[2] B.P. Abbott et al., LIGO Scientific Collaboration and Virgo Collaboration, "GW170817: Observation of Gravitational Waves from a Binary Neutron Star Inspiral,” Phys. Rev. Lett. 119 (16), 161101 (2017).

[3] B. P. Abbott et al., "Exploring the Sensitivity of Next Generation Gravitational Wave Detectors," Class. Quantum Grav. 34(4), 044001 (2017).

[4] ET Science Team, "Einstein gravitational wave Telescope Conceptual Design Study," available at http://www.et-gw.eu/, Date of access: Jan. 08 2018, ET-0106A-10, (2011).

[5] A. Schroeter et al., "On the mechanical quality factors of cryogenic test masses from fused silica and crystalline quartz”, Class. Quantum Grav, LIGO-P070100-00-Z (2007).

[6] M. D. Mermelstein, K. Brar, M. J. Andrejco, A. D. Yablon, M. Fishteyn, C. Headley III, and D. J. DiGiovanni, "All-fiber 194W single-frequency single-mode Yb-doped master-oscillator power amplifier," Proc. SPIE, 6873 (2008).

[7] P. Ma, P. Zhou, Y. Ma, R. Su, X. Xu, and Z. Liu, "Single frequency 332 W, linearly polarized Yb-doped all-fiber amplifier with near diffraction-limited beam quality," Appl. Opt. 52, 4854-4857 (2013).

[8] L. Huang, H. Wu, R. Li, L. Li, P. Ma, X. Wang, J. Leng, and P. Zhou, "414 W near-diffraction-limited allfiberized single-frequency polarization-maintained fiber amplifier," Opt. Lett. 42, 1-4 (2017). 
[9] T. Theeg, H. Sayinc, J. Neumann, L. Overmeyer, D. Kracht, "Fiber integrated Ytterbium single frequency amplifier with a measured fundamental mode power content of $91 \%$ at 215 W“, Workshop on Specialty Optical Fibers and their Applications (Optical Society of America), paper W3.33 (2013).

[10] O. de Varona et al., "Single-frequency fiber amplifier at $1.5 \mu \mathrm{m}$ with $100 \mathrm{~W}$ in the linearly-polarized TEM 00 mode for next-generation gravitational wave detectors", Opt. Express 25, 24880-24892 (2017).

[11] T. Theeg et al., "All-Fiber Counter-Propagation Pumped Single Frequency Amplifier Stage With 300 W Output Power", IEEE Photonics Technology Letters 24 (20), 1864-1867 (2012).

[12] T. Theeg et al., "Pump and signal combiner for bi-directional pumping of all-fiber lasers and amplifiers," Opt. Express 20 (27), 28125 (2012).

[13] V. I. Kovalev, R. G. Harrison, "Suppression of stimulated Brillouin scattering in high-power singlefrequency fiber amplifiers", Opt. Lett. 31, 161-163 (2006).

[14] P. Kwee, F. Seifert, B.Willke, K. Danzmann, "Laser beam quality and pointing measurements with an optical resonator,“ Rev. Sci. Instrum. 78 (7), 073103 (2007).

[15] D. Creeden, H. Pretorius, J. Limongelli, and S. D. Setzler, "Single frequency $1560 \mathrm{~nm} \mathrm{Er:Yb} \mathrm{fiber}$ amplifier with 207 W output power and 50.5 \% slope efficiency," Proc. SPIE 9728, 97282L (2016).

[16] M. Steinke et al., "Single-Frequency Fiber Amplifiers for Next-Generation Gravitational Wave Detectors", IEEE J. Sel. Top. Quantum Electron. 24(3), in press (2018).

[17] M. Steinke, J. Neumann, D. Kracht, and P. Wessels, "Gain dynamics in $\mathrm{Er}^{3+}: \mathrm{Yb}^{3+}$ co-doped fiber amplifiers," Opt. Express 23(11), 14946-14959 (2015).

[18] M. Wysmolek, T. Theeg, H. Sayinc, J. Neumann, and D. Kracht, “All-fibre high power pump stripper manufactured by CO2 laser micro-structuring,” LASE Fiber Lasers X, 8601-21 (2013). 